\title{
Numerical Investigation with Stability Convergence Analysis of Chemically Hydromagnetic Casson Nanofluid Flow in the Effects of Thermophoresis and Brownian Motion
}

\author{
Munmun Mondal $^{1}$, Rajib Biswas ${ }^{2 *}$, Kazi Shanchia ${ }^{1}$, Mehedy Hasan $^{1}$, Sarder F. Ahmmed $^{1}$ \\ ${ }^{1}$ Mathematics Discipline, Khulna University, Khulna-9208, Bangladesh \\ ${ }^{2}$ Department of Mathematics, Bangladesh University, Dhaka-1207, Bangladesh
}

Corresponding Author Email: rajibkumath11@gmail.com

https://doi.org/10.18280/ijht.370107

Received: 16 March 2018

Accepted: 23 July 2018

\section{Keywords:}

Casson nanofluid, EFDM, radiation, chemical reaction, $M H D$, porous medium

\begin{abstract}
In this evaluation, a numerical exploration is inscribed on MHD unsteady heat and mass transfer of Casson nanofluid flow where variable thermal conductivity, radiation and heat absorption are counterfeited. The model is implemented for the nanofluid which annexes the impression of thermophoresis and Brownian motion. The governing models are metamorphosed as a dimensionless silhouette by the renovation rule of mathematics and the procured dimensionless couple of partial differential equations (PDEs) are elucidated by utilizing explicit finite difference method (EFDM). The numerical aggregates are enumerated for the miscellaneous dimensionless parameters on velocity, temperature and concentration profiles along with the skin friction, Nusselt number and Sherwood number with distinct time interims. ForTran programing language is manipulated as the predominated software for executing the numerical values. Also, by wielding the initial boundary conditions $U=T=C=0$, $\Delta \mathrm{X}=0.83$ and $\Delta \mathrm{Y}=0.50$ for $\Delta \tau=0.0005$, convergence criteria are exhibited with $\mathrm{Sc} \geq 0.18$ and $\mathrm{Le} \geq 0.20$. At the end, the prevailed culminations are illustrated and perused after the stability convergence test (SCT) by manoeuvring graphics software tecplot- 9 and streamlines and isotherms are delineated.
\end{abstract}

\section{INTRODUCTION}

Casson fluid is a non-Newtonian shear thinning fluid appending with infinite viscosity at zero shear rates. Pragmatic implementations of Casson fluid comprise in the sector of petroleum drilling, polymer engineering, certain separation processes, manufacturing of foods and paper. In addition, biological, chemical, medical and engineering applications are vitally pivotal affiliated to the Casson fluid. Distinct inquisitors have implemented their fact-finding pursuit in this direction enlightening the consequences of numerous parameters on the flow of the Casson fluid. Unsteady Casson nanofluid flow over a stretching sheet have been appraised by Oyelakin et al. [1]. Kataria and Patel [2] have scrutinized the Soret and heat generation sequels on MHD Casson fluid flow. The repercussions on magnetic field in squeezing flow of a Casson fluid have been probed by Ahmed et al. [3]. Furthermore, Mahanta et al. [4] perused the 3D Casson fluid flow. In very recent time, Afikuzzaman et al. [5]; Biswas et al. [6]; Afikuzzaman et al. [7] and Wahiduzzaman et al. [8] have numerically evaluated unsteady MHD Casson fluid flow. Also, Casson nanofluid has been contemplated by abundant scrutinisers such as: Biswas et al. [9]; Khalid et al. [10]; Ali et al. [11] and Ghadikolaei et al. [12].

A nanofluid is a fluid, mingle with nanometer-sized particles typically made of metals, oxides and carbides. In 1995, the abstraction of nanofluids has been initiated by Chai et al. [13], where the breach of nanaparticles in a common base fluids include water, ethylene glycol and oil has proffered. Nanofluids have some substantial properties that assemble them potentially convenient in many exertions in heat transfer, including microelectronics, fuel cells, and pharmaceutical processes. Ganga et al. [14] delineated the MHD flow of Boungiorno model nanofluid over a vertical plate. In addition, optimal interpretation of nonlinear heat and mass transfer in a two-layer flow with nano-Eyring-Powell fluid was assembled by Khan et al. [15]. Denouements of magnetic field and radiation absorption on mixed convective Jeffrey nanofluid flow have been dispatched by Biswas et al. [16]. Explicit numerical study of unsteady hydromagnetic mixed convective nanofluid flow elaborated by Beg et al. [17]. Heat transfer of MHD nanofluid in disparate view have been propounded by multifarious assessors analogous as Dogonchi et al. [18]; Dogonchi et al. [19]; Ahmmed et al. [20]; Leekea et al. [21]; Uddin et al. [22]; Bilal et al. [23] and Arani et al. [24].

In the past several decades, the evaluation of magnetohydrodynamic (MHD) mass transfer flow has procured apocalyptic to profuse scholar's due to its diverse industrial applications such as the cooling of reactors, power generators, electrostatic precipitation, polymer technology petroleum industry, aerodynamics heating, petroleum industry, the operations of MHD accelerators, fluid droplets sprays, agriculture, geophysics, metrology, petro-chemical industry and so on. Heat and mass transfer sequels on MHD natural convective flow was implemented by Murthy et al. [25]. Das et al. [26] have instigated magnetohydrodynamic mixed convective slip flow. Convective heat transfer and MHD viscoelastic nanofluid flow has been actuated by Shit et al. [27]. In contemporary epoch, MHD has become a great fact-finding argument of computational fluid dynamics (CFD) which contrived by multitudinous scientists commensurate as Ibrahim [28]; Rani and Kim [29]; Animasaun and Oyem [30] 
and Lavanya and Ratnam [31].

Homogeneous to MHD deportment, chemical reaction is a stratagem that assists to the transformation of one set of chemical substances to another at a fixed temperature and concentration. Heat and mass transfer on MHD mixed convection axisymmetric chemically reactive flow of Maxwell fluid flow was evoked by Khan et al. [32]. Also, Tripathy et al. [33] have illustrated the chemical reaction effect. Here, authors have renovated their governing equations into a twopoint boundary value predicament manipulating similarity variables and then elucidated numerically by forth order Runge-Kutta method with shooting modus operandi.

Electromagnetic radiation has anonymous conventional characteristics depending on the frequency and wavelengths of the radiation simulates a vital role in industrial and technological areas such as various space vehicles, satellites, missiles, aircraft, gas turbines and nuclear reactors. Das et al. [34] perused Hall denouements with radiative heat transfer past a porous plate. They disentangled their governing equations by wielding fourth order Runge-Kutta- Fehlberg method with the shooting technique. Thermal radiation culmination was elaborated by Dogonchi by et al. [35]. In addition, combined electrical MHD heat transfer with radiative and viscous dissipation out-turns have been unveiled by Hsiao [36]; Biswas et al. [37]; Ahmmed et al. [38] and Biswas et al. [39].

The intention of the contemporaneous interpretation is to evaluate the sequels of MHD unsteady heat and mass transfer of Casson nanofluid flow past an exponentially accelerated plate. The consecutive Casson nanofluid model is reassessed to affix the consequence of thermophoresis and Brownian motion. We have pivoted the culminations of heterogeneous parameters such as of Casson parameter, permeability of porous medium, radiation parameter, Lewis number, thermophoresis parameter, Brownian motion parameter, Soret number, magnetic parameter, Schmidt number, Prandtl number, Heat source parameter, chemical reaction parameter, Grashof number and modified Grashof number. Furthermore, we have evaluated the thermal and momentum boundary layer thickness with isotherms and streamlines analysis. Finally, the present outcomes have been analogized with the foregoing ramifications.

\section{MATHEMATICAL ANALYSIS}

The unsteady two dimensional free convection Casson nanofluid flow of an electrically conducting viscous incompressible fluid through a vertical porous permeable plate in the manifestation of thermal radiation, thermal diffusion and chemical reaction are enumerated with the $\mathrm{x}$-axis is contemplated along the plate in the precipitously up sloping orientation and the $y$-axis is stipulated normal to the plate. A uniform magnetic field strength $\mathrm{B}_{0}$ is implemented across to the flow direction. It is appraised that the temperature of the wall is $T_{w}$ and concentration at the plate is $C_{w}$ at initially which are constant but at time $t>0$. The plate is precipitated exponentially with a velocity $\mathrm{u}=\mathrm{U}_{0} \exp \left(\mathrm{a}^{\prime} \mathrm{t}\right)$ in its own plane and the temperature and concentration level of the plate are elevated exponentially to $\mathrm{T}_{\mathrm{w}}\left(=\mathrm{T}_{\infty}\right)$ and $\mathrm{C}_{\mathrm{w}}\left(=\mathrm{C}_{\infty}\right)$ respectively with time $t$. The physical configuration and coordinate system of this present model is unveiled in Figure 1.

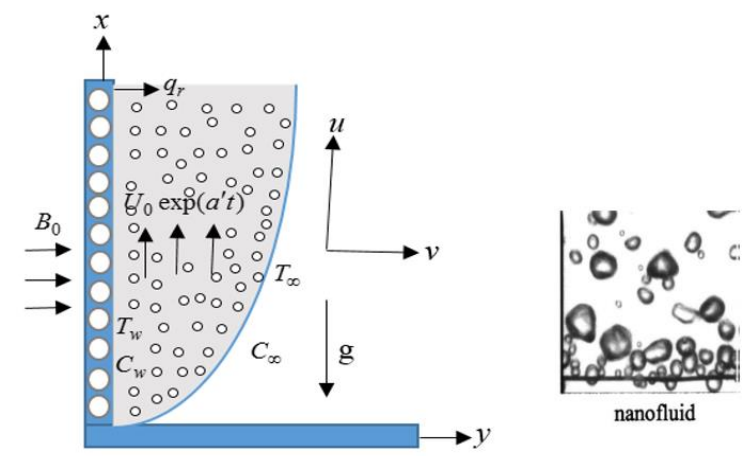

Figure 1. Physical configuration

According to Oyelakin et al. [1] and Biswas et al. [9], the rheological equation of Casson fluid flow has been contemplated as:

$\tau_{i j}=2\left(\mu_{b}+\frac{P_{y}}{\sqrt{2 \pi}}\right) e_{i j} \quad$ when $\pi>\pi_{c}$

$\tau_{i j}=2\left(\mu_{b}+\frac{P_{y}}{\sqrt{2 \pi_{c}}}\right) e_{i j} \quad$ when $\pi<\pi_{c}$

where, $\tau_{\mathrm{ij}}$ is the component of stress tension, $\pi$ is the product of the component of deformation rate with itself, $e_{i j}$ is $(i, j)$ th component of the deformation rate, $\pi_{\mathrm{c}}$ is critical value based on the non-Newtonian model and $\mathrm{P}_{\mathrm{y}}$ is comprehended as yield stress of the fluid which is verbalized as:

$P_{y}=\frac{\mu_{b} \sqrt{2 \pi}}{\beta}$

where, $\mu_{\mathrm{b}}=$ plastic dynamic viscosity of Non-Newtonian fluid. In a case of Casson fluid flow, where $\pi>\pi_{c}$, therefore

$\mu=\mu_{b}+\frac{P_{y}}{\sqrt{2 \pi}}$ or, $\mu=\mu_{b}+\frac{\frac{\mu_{b} \sqrt{2 \pi}}{\beta}}{\sqrt{2 \pi}}$ or, $v=\frac{\mu_{b}}{\rho}\left(1+\frac{1}{\beta}\right)$

Under these speculations, the pivotal dimensional governing equations with boundary conditions can be asserted as:

$$
\begin{aligned}
& \frac{\partial u}{\partial x}+\frac{\partial v}{\partial y}=0 \\
& \frac{\partial u}{\partial t}+u \frac{\partial u}{\partial x}+v \frac{\partial u}{\partial y}=v\left(1+\frac{1}{\beta}\right) \frac{\partial^{2} u}{\partial y^{2}}+g \beta_{T}\left(T-T_{\infty}\right) \\
& +g \beta_{C}\left(C-C_{\infty}\right)-\left(1+\frac{1}{\beta}\right) \frac{v}{k^{*}} u-\frac{\sigma B_{0}^{2} u}{\rho} \\
& \frac{\partial T}{\partial t}+u \frac{\partial T}{\partial x}+v \frac{\partial T}{\partial y}=\frac{1}{\rho C_{p}} \frac{\partial}{\partial y}\left(k \frac{\partial T}{\partial y}\right)-\frac{1}{\rho C_{p}} \frac{\partial q_{r}}{\partial y} \\
& +\tau^{\prime}\left\{D_{B}\left(\frac{\partial C}{\partial y} \frac{\partial T}{\partial y}\right)+\frac{D_{T}}{T_{\infty}}\left(\frac{\partial T}{\partial y}\right)^{2}\right\}+\frac{Q}{\rho C_{p}}\left(T-T_{\infty}\right)
\end{aligned}
$$


$\frac{\partial C}{\partial t}+u \frac{\partial C}{\partial x}+v \frac{\partial C}{\partial y}=D_{m} \frac{\partial^{2} C}{\partial y^{2}}-K_{1}\left(C-C_{\infty}\right)$

$+D_{B} \frac{\partial^{2} C}{\partial y^{2}}+\frac{D_{T}}{T_{\infty}} \frac{\partial^{2} T}{\partial y^{2}}+\frac{D_{m} K_{T}}{T_{m}} \frac{\partial^{2} T}{\partial y^{2}}$

The corresponding boundary conditions are:

$$
\begin{aligned}
& t \leq 0, u=0, v=0, T=T_{w}, C=C_{w} \quad \text { for all } y \\
& t>0, u=U_{0} e^{\left(a^{\prime} t\right)}, v=0, T=T_{\infty}+\left(T_{w}-T_{\infty}\right) e^{\left(\frac{U_{0}^{2}}{v} t\right)} \\
& C=C_{\infty}+\left(C_{w}-C_{\infty}\right) e^{\left(\frac{U_{0}^{2}}{v} t\right)} \quad \text { at } y=0 \\
& \left.u=0, v=0, T \rightarrow T_{\infty}, C \rightarrow C_{\infty} \quad \text { as } y \rightarrow \infty\right)
\end{aligned}
$$

where, $\tau^{\prime}=(\rho c) p /(\rho c) f$ is the ratio of the heat capacity of the nanoparticle material and the heat capacity of the fluid. The dimensionless governing equations have been solicited by appertaining the subsequent dimensionless parameters and variables

$$
\begin{aligned}
& U=\frac{u}{U_{0}} ; V=\frac{v}{U_{0}} ; Y=\frac{y U_{0}}{v} ; X=\frac{x U_{0}}{v} ; \tau=\frac{t U_{0}^{2}}{v} ; \\
& T=T_{\infty}+\bar{T}\left(T_{w}-T_{\infty}\right) ; C=C_{\infty}+\bar{C}\left(C_{w}-C_{\infty}\right) ; \\
& \operatorname{Pr}=\frac{v \rho C_{p}}{k} ; G r=\frac{v g \beta_{T}\left(T_{w}-T_{\infty}\right)}{U_{0}^{3}} ; G m=\frac{v g \beta_{c}\left(C_{w}-C_{\infty}\right)}{U_{0}^{3}} \\
& S=\frac{Q v}{\rho C_{p} U_{0}^{2}} ; M=\frac{\sigma B_{0}^{2} v}{\rho U_{0}^{2}} ; L e=\frac{v}{D_{B}} ; \\
& S c=\frac{v}{D_{m}} ; S r=\frac{D_{m} K_{T}}{T_{m} v} \frac{\left(T_{w}-T_{\infty}\right)}{\left(C_{w}-C_{\infty}\right)} \\
& \gamma=\frac{K_{1} v}{U_{0}^{2}} ; R a=\frac{4 \sigma^{\prime} T_{\infty}^{3}}{k^{\prime} k} ; K p=\frac{v^{2}}{k^{*} U_{0}^{2}} ; a=\frac{a_{1}^{\prime} v}{U_{0}^{2}} \\
& N t=\frac{\tau^{\prime} D_{T}\left(T_{w}-T_{\infty}\right)}{T_{\infty} v} ; N b=\frac{\tau^{\prime} D_{B}\left(C_{w}-C_{\infty}\right)}{v}
\end{aligned}
$$

Therefore, the dimensionless equations are:

$$
\frac{\partial U}{\partial X}+\frac{\partial V}{\partial Y}=0
$$

$\frac{\partial U}{\partial \tau}+U \frac{\partial U}{\partial X}+V \frac{\partial U}{\partial Y}=\left(1+\frac{1}{\beta}\right) \frac{\partial^{2} U}{\partial Y^{2}}+G r \bar{T}$

$+G m \bar{C}-\left(1+\frac{1}{\beta}\right) K p U-M U$

$$
\begin{aligned}
& \frac{\partial \bar{T}}{\partial \tau}+U \frac{\partial \bar{T}}{\partial X}+V \frac{\partial \bar{T}}{\partial Y}=\frac{1}{\operatorname{Pr}}\left\{\frac{\lambda}{1+\lambda \bar{T}}\left(\frac{\partial \bar{T}}{\partial Y}\right)^{2}\right\}+\frac{1}{\operatorname{Pr}} \\
& \left(1+\frac{4}{3} R a\right) \frac{\partial^{2} \bar{T}}{\partial Y^{2}}+S \bar{T}+N t\left(\frac{\partial \bar{T}}{\partial Y}\right)^{2}+N b \frac{\partial \bar{C}}{\partial Y} \frac{\partial \bar{T}}{\partial Y} \\
& \frac{\partial \bar{C}}{\partial \tau}+U \frac{\partial \bar{C}}{\partial X}+V \frac{\partial \bar{C}}{\partial Y}=\frac{1}{S c} \frac{\partial^{2} \bar{C}}{\partial Y^{2}}-\gamma \bar{C} \\
& +\frac{1}{L e} \frac{\partial^{2} \bar{C}}{\partial Y^{2}}+\frac{N t}{N b L e} \frac{\partial^{2} \bar{T}}{\partial Y^{2}}+S r \frac{\partial^{2} \bar{T}}{\partial Y^{2}}
\end{aligned}
$$

The boundary conditions can be elicited as:

$$
\left.\begin{array}{c}
\tau>0, U=\mathrm{e}^{(a \tau)}, V=0, \bar{T}=\mathrm{e}^{(\tau)}, \bar{C}=\mathrm{e}^{(\tau)} \text { at } Y=0 \\
U=0, \bar{T} \rightarrow 0, \bar{C} \rightarrow 0 \quad \text { as } Y \rightarrow \infty
\end{array}\right\}
$$

The skin friction coefficient, Nusselt number and Sherwood number are evolved as:

$$
\begin{aligned}
& C_{f}=-\frac{1}{2 \sqrt{2}}\left(G_{r}\right)^{-\frac{3}{4}}\left(\frac{\partial U}{\partial Y}\right)_{Y=0} \\
& N_{u}=\frac{1}{\sqrt{2}}\left(G_{r}\right)^{-\frac{3}{4}}\left(\frac{\partial \bar{T}}{\partial Y}\right)_{Y=0} \\
& S_{h}=\frac{1}{\sqrt{2}}\left(G_{r}\right)^{-\frac{3}{4}}\left(\frac{\partial \bar{C}}{\partial Y}\right)_{Y=0}
\end{aligned}
$$

The stream function $\psi(\mathrm{X}, \mathrm{Y})$ with the velocity components are devised as:

$$
U=\frac{\partial \psi}{\partial Y}, V=-\frac{\partial \psi}{\partial X}
$$

\section{CALCULATION TECHNIQUE}

An explicit finite difference scheme has been reconnoitred for deciphering the equations 14-17, because of that a rectangular region is ascertained where $\mathrm{X}$ - axis is pondered along the plate and $\mathrm{Y}$-axis is normal to the plate. Here, plate of height is $X_{\max }(=125)$ and $Y_{\max }(=125)$. Also, grid spacing are esteemed $m=150$ and $n=300$ which are spectacled in Figure 1(a).

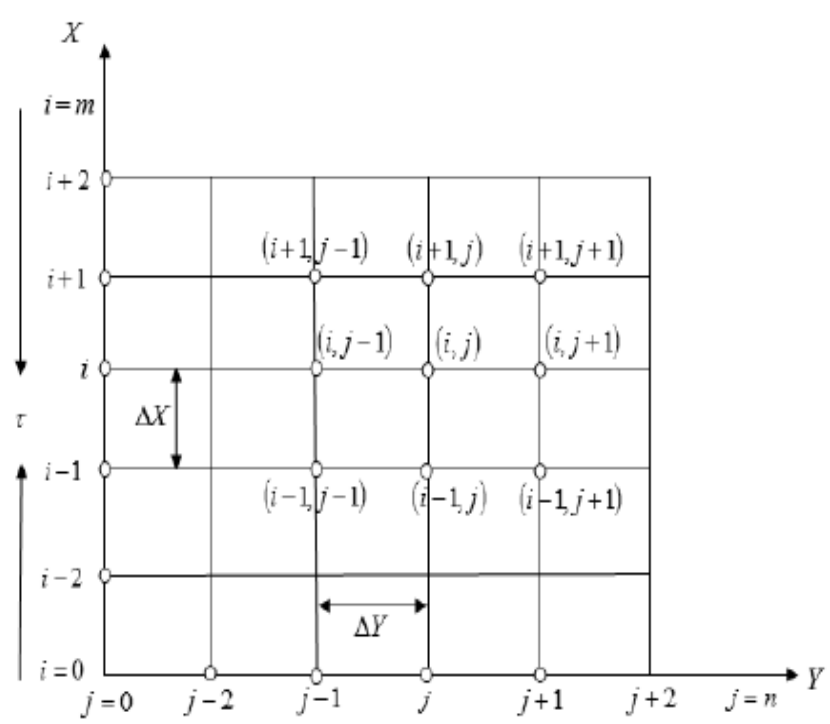

Figure 1(a). The finite difference space grid

The constant mash size is wangled $\Delta X=0.83(0 \leq x \leq$ 125); $\Delta Y=0.50(0 \leq y \leq 125)$ with the smaller time-step, $\Delta \tau=0.0005$. Now,

$\frac{U_{i, j}-U_{i-1, j}}{\Delta X}+\frac{V_{i, j}-V_{i, j-1}}{\Delta Y}=0$ 


$$
\begin{aligned}
& \frac{U_{i, j}^{\prime}-U_{i, j}}{\Delta \tau}+U_{i, j}\left(\frac{U_{i, j}-U_{i-1, j}}{\Delta X}\right)+V_{i, j}\left(\frac{U_{i, j+1}-U_{i, j}}{\Delta \mathrm{Y}}\right) \\
& =\left(1+\frac{1}{\beta}\right)\left(\frac{U_{i, j+1}-2 U_{i, j}+U_{i, j-1}}{(\Delta \mathrm{Y})^{2}}\right)+G r \bar{T}_{i, j} \\
& +G m \bar{C}_{i, j}-\left(1+\frac{1}{\beta}\right) k p U_{i, j}-M U_{i, j} \\
& \frac{\bar{T}_{i, j}^{\prime}-\bar{T}_{i, j}}{\Delta \tau}+U_{i, j} \frac{\bar{T}_{i, j}-\bar{T}_{i-1, j}}{\Delta X}+V_{i, j} \frac{\bar{T}_{i, j+1}-\bar{T}_{i, j}}{\Delta Y} \\
& =\frac{1}{\operatorname{Pr}}\left(1+\frac{4}{3} R a\right) \frac{\bar{T}_{i, j+1}-2 \bar{T}_{i, j}+\bar{T}_{i, j-1}}{(\Delta Y)^{2}}+S \bar{T}_{i, j} \\
& +\frac{1}{\operatorname{Pr}}\left(\frac{\lambda}{1+\lambda \bar{T}_{i, j}}\right)\left(\frac{\bar{T}_{i, j+1}-\bar{T}_{i, j}}{\Delta \mathrm{Y}}\right)^{2}+N b \\
& \left(\frac{\bar{C}_{i, j+1}-\bar{C}_{i, j}}{\Delta Y}\right)\left(\frac{\bar{T}_{i, j+1}-\bar{T}_{i, j}}{\Delta Y}\right)+N t\left(\frac{\bar{T}_{i, j+1}-\bar{T}_{i, j}}{\Delta Y}\right)^{2} \\
& \frac{\bar{C}_{i, j}^{\prime}-C_{i, j}}{\Delta \tau}+U_{i, j} \frac{\bar{C}_{i, j}-\bar{C}_{i-1, j}}{\Delta X}+V_{i, j} \frac{\bar{C}_{i, j+1}-\bar{C}_{i, j}}{\Delta Y} \\
& =\left(\frac{1}{S c}+\frac{1}{L e}\right) \frac{\bar{C}_{i, j+1}-2 \bar{C}_{i, j}+\bar{C}_{i, j-1}}{(\Delta Y)^{2}}-\gamma \bar{C}_{i, j} \\
& +\left(S r+\frac{N_{t}}{N b L e}\right) \frac{\bar{T}_{i, j+1}-2 \bar{T}_{i, j}+\bar{T}_{i, j-1}}{(\Delta Y)^{2}}
\end{aligned}
$$

The intermeddle boundary periphery in explicit form as:

$$
\left.\begin{array}{c}
\tau>0, U_{i, 0}^{n}=\mathrm{e}^{(a n \Delta \tau)}, V_{i, 0}^{n}=0, \bar{T}_{i, 0}^{n}=\mathrm{e}^{(n \Delta \tau)}, \\
\bar{C}_{i, 0}^{n}=\mathrm{e}^{(n \Delta \tau)} \quad \text { at } \mathrm{Y}=0 \\
U_{i, L}^{n}=0, \bar{T}_{i, L}^{n} \rightarrow 0, \bar{C}_{i, L}^{n} \rightarrow 0 \text { where } L \rightarrow \infty
\end{array}\right\}
$$

Here $\mathrm{i}$ and $\mathrm{j}$ designate to the mesh points with $\mathrm{X}$ and $\mathrm{Y}$ coordinate respectively and the superscripts $n$ connote a value of time, $\tau=n \Delta \tau$ where, $n=0,1,2 \ldots$.

\section{STABILITY AND CONVERGENCE ANALYSIS}

The exploration persists deficient without the stability and convergence test (SCT) as long as the finite difference scheme has been exerted. For the explicit mesh sizes, the stability criteria of the scheme has been actuated as follows:

The equation (14) has been eradicated because $\Delta \tau$ does not transpire in it. The quotidian terms of the Fourier expansion for $U, \bar{T}$ and $\bar{C}$ at a logically time $\tau=0$ are all $\mathrm{e}^{\mathrm{i} \alpha \mathrm{X}} \mathrm{e}^{\mathrm{i} \beta \mathrm{Y}}$ aloof form a constant, where $i=\sqrt{-1}$. At a time $\tau$, these terms become:

$$
\left.\begin{array}{ll}
U: & A(\tau) e^{i \alpha X} e^{i \beta Y} \\
\bar{T}: & C(\tau) e^{i \alpha X} e^{i \beta Y} \\
\bar{C}: & D(\tau) e^{i \alpha X} e^{i \beta Y}
\end{array}\right\}
$$

After the time step,

$$
\left.\begin{array}{ll}
U: & A^{\prime}(\tau) e^{i \alpha X} e^{i \beta Y} \\
\bar{T}: & C^{\prime}(\tau) e^{i \alpha X} e^{i \beta Y} \\
\bar{C}: & D^{\prime}(\tau) e^{i \alpha X} e^{i \beta Y}
\end{array}\right\}
$$

By cast away the above values the following palliation equations are erected as:

$$
\begin{aligned}
& \frac{\left(A^{\prime}-A\right) e^{i \alpha X} e^{i \beta Y}}{\Delta \tau}+U \frac{A e^{i \alpha X} e^{i \beta Y}\left(1-e^{-i \alpha \Delta X}\right)}{\Delta X} \\
& +V \frac{A e^{i \alpha X} e^{i \beta Y}\left(e^{i \beta \Delta Y}-1\right)}{\Delta Y}=\left(1+\frac{1}{\beta}\right) \\
& \frac{2 A e^{i \alpha X} e^{i \beta Y}(\cos \beta \Delta Y-1)}{(\Delta Y)^{2}}+G r C e^{i \alpha X} e^{i \beta Y}+G m D e \\
& i \alpha X e^{i \beta Y}-\left(1+\frac{1}{\beta}\right) K p A e^{i \alpha X} e^{i \beta Y}-M A e^{i \alpha X} e^{i \beta Y} \\
& \frac{\left(C^{\prime}-C\right) e^{i \alpha X} e^{i \beta Y}}{\Delta \tau}+U \frac{C e^{i \alpha X} e^{i \beta Y}\left(1-e^{-i \alpha \Delta X}\right)}{\Delta X} \\
& +V \frac{C e^{i \alpha X} e^{i \beta Y}\left(e^{i \beta \Delta Y}-1\right)}{\Delta Y}=\frac{1}{\operatorname{Pr}}\left(1+\frac{4}{3} R a\right) \\
& \frac{2 C e^{i \alpha X} e^{i \beta Y}(\cos \beta \Delta Y-1)}{(\Delta Y)^{2}}+S C e^{i \alpha X} e^{i \beta Y} \\
& +N b \frac{D e^{i \alpha X} e^{i \beta Y}\left(1-e^{-i \alpha \Delta X}\right)}{\Delta X} \frac{C e^{i \alpha X} e^{i \beta Y}\left(e^{i \beta \Delta Y}-1\right)}{\Delta Y} \\
& \frac{\left(D^{\prime}-D\right) e^{i \alpha X} e^{i \beta Y}}{\Delta \tau}+U \frac{D e^{i \alpha X} e^{i \beta Y}\left(1-e^{-i \alpha \Delta X}\right)}{\Delta X} \\
& +V \frac{D e^{i \alpha X} e^{i \beta Y}\left(e^{i \beta \Delta Y}-1\right)}{\Delta Y}=\left(\frac{1}{S c}+\frac{1}{L e}\right) \\
& \left.+\left(\frac{2 D e^{i \alpha X} e^{i \beta Y}(\cos \beta \Delta Y-1)}{(\Delta Y)^{2}}\right] \frac{N t}{N b L e}\right)\left[\frac{2 C e^{i \alpha X} e^{i \beta Y}(\cos \beta \Delta Y-1)}{(\Delta Y)^{2}}\right] \\
& -\gamma D e^{i \alpha X} e^{i \beta Y}
\end{aligned}
$$

The overhead equations are revealed as

$$
\begin{aligned}
& A^{\prime}=A_{1} A+A_{2} C+A_{3} D \\
& C^{\prime}=A_{4} C \\
& D^{\prime}=A_{5} D+A_{6} C
\end{aligned}
$$

where,

$$
\begin{aligned}
& A_{1}=1+\left(1+\frac{1}{\beta}\right) \frac{2 \Delta \tau}{(\Delta Y)^{2}}(\cos \beta \Delta Y-1)-U \frac{\Delta \tau}{\Delta X} \\
& \left(1-e^{-i \alpha \Delta X}\right)-V \frac{\Delta \tau}{\Delta Y}\left(e^{i \beta \Delta Y}-1\right) \\
& -\left(1+\frac{1}{\beta}\right) K p \Delta \tau-M \Delta \tau \\
& A_{2}=G r \Delta \tau ; A_{3}=G m \Delta \tau
\end{aligned}
$$


$A_{4}=1+\frac{1}{\operatorname{Pr}}\left(1+\frac{4}{3} R a\right) \frac{2 \Delta \tau}{(\Delta Y)^{2}}(\cos \beta \Delta Y-1)$

$-U \frac{\Delta \tau}{\Delta X}\left(1-e^{-i \alpha \Delta X}\right)-V \frac{\Delta \tau}{\Delta Y}\left(e^{i \beta \Delta Y}-1\right)+S \Delta \tau$

$+\frac{1}{\operatorname{Pr}}\left(\frac{\lambda}{1+\lambda \bar{C}}\right) \frac{2 \Delta \tau}{(\Delta Y)^{2}}(\cos \beta \Delta Y-1)$

$-U \frac{\Delta \tau}{\Delta X}\left(1-e^{-i \alpha \Delta X}\right)-V \frac{\Delta \tau}{\Delta Y}\left(e^{i \beta \Delta Y}-1\right)$

$+S \Delta \tau$

$A_{5}=1+\left(\frac{1}{S c}+\frac{1}{L e}\right) \frac{2 \Delta \tau}{(\Delta Y)^{2}}(\cos \beta \Delta Y-1)$

$-U \frac{\Delta \tau}{\Delta X}\left(1-e^{-i \alpha \Delta X}\right)-V \frac{\Delta \tau}{\Delta Y}\left(e^{i \beta \Delta Y}-1\right)-\gamma \Delta \tau$

$A_{6}=\left(S r+\frac{N t}{N b L e}\right) \frac{2 \Delta \tau}{(\Delta Y)^{2}}(\cos \beta \Delta Y-1)$

Therefore the matrix form is:

$\left[\begin{array}{l}A^{\prime} \\ C^{\prime} \\ D^{\prime}\end{array}\right]=\left[\begin{array}{ccc}A_{1} & A_{2} & A_{3} \\ 0 & A_{4} & 0 \\ 0 & A_{6} & A_{5}\end{array}\right]\left[\begin{array}{l}A \\ B \\ C\end{array}\right]$

Therefore, $\eta^{\prime}=T^{\prime} \eta$ where: $\eta^{\prime}=\left[\begin{array}{l}A^{\prime} \\ C^{\prime} \\ D^{\prime}\end{array}\right]$;

$T^{\prime}=\left[\begin{array}{ccc}A_{1} & A_{2} & A_{3} \\ 0 & A_{4} & 0 \\ 0 & A_{6} & A_{5}\end{array}\right] ; \quad \eta=\left[\begin{array}{l}A \\ B \\ C\end{array}\right]$

This is a fourth order square matrix. So, it is very hard to find out the eigenvalues and $\Delta \tau$ is very compact in EFDM, i.e. $\Delta \tau \rightarrow 0$. Under this appraisal, $\mathrm{A}_{2} \rightarrow 0, \mathrm{~A}_{3} \rightarrow 0$ and $\mathrm{A}_{6} \rightarrow 0$.

Therefore, $T^{\prime}=\left[\begin{array}{ccc}A_{1} & 0 & 0 \\ 0 & A_{4} & 0 \\ 0 & 0 & A_{5}\end{array}\right]$;

Therefore the eigenvalues are retrieved as $\lambda_{1}=\mathrm{A}_{1}, \lambda_{2}=\mathrm{A}_{4}$ and $\lambda_{3}=\mathrm{A}_{5}$ such that $\left|A_{1}\right| \leq 1\left|A_{4}\right| \leq 1$ and $\left|A_{5}\right| \leq 1$ and convinced admissible values are $\mathrm{A}_{1}=-1, \mathrm{~A}_{4}=-1$ and $\mathrm{A}_{5}=-1$.

So, the stability conditions are:

$\left(1+\frac{1}{\beta}\right) \frac{2 \Delta \tau}{(\Delta Y)^{2}}+\frac{M \Delta \tau}{2}+U \frac{\Delta \tau}{\Delta X}+|-V| \frac{\Delta \tau}{\Delta Y}+$

$\left(1+\frac{1}{\beta}\right) \frac{K p \Delta \tau}{2} \leq 1$

$\frac{1}{\operatorname{Pr}}\left(1+\frac{4}{3} R a\right) \frac{2 \Delta \tau}{(\Delta Y)^{2}}+\left(\frac{1}{\operatorname{Pr}}+N t\right)\left(\frac{\Delta \tau}{\Delta Y}\right)^{2}$

$+U \frac{\Delta \tau}{\Delta X}+|-V| \frac{\Delta \tau}{\Delta Y}-\frac{S \Delta \tau}{2} \leq 1$

$\left(\frac{1}{S c}+\frac{1}{L e}\right) \frac{2 \Delta \tau}{(\Delta Y)^{2}}+\frac{\gamma \Delta \tau}{2}+U \frac{\Delta \tau}{\Delta X}+|-V| \frac{\Delta \tau}{\Delta Y} \leq 1$
Exploiting basic agreement, $\mathrm{U}=\mathrm{V}=\mathrm{T}=\mathrm{C}=0$ at $\tau=0$ the convergence criteria of the existent problem are retrieved as $\mathrm{Sc} \geq 0.18$ and $\mathrm{Le} \geq 0.20$.

\section{RESULTS AND DISCUSSION}

The numerical computations have been manipulated for the disparate values of miscellaneous parameters by an explicit finite difference method (EFDM) for the aspiration of scrutinizing the upshots of the contemporary work. The physical stipulation of the contemporaneous model such that velocity, temperature, concentration, skin friction coefficient, Nusselt number, Sherwood number, streamlines and isotherms lines has been procured for distinct parameters which are indicated in Figures 2 to 23. In order to prevail the numerical results, the succeeding values of default parameter are deputed as: $\mathrm{M}=1.0, \mathrm{Pr}=0.71, \mathrm{Sc}=0.22, \mathrm{Ra}=0.50, \quad \beta=0.40, \mathrm{~S}=0.1$, $\mathrm{Nb}=0.5, \mathrm{Nt}=0.8, \mathrm{Le}=2.5, \mathrm{Sr}=1, \mathrm{Gm}=5, \lambda=2, \mathrm{Gr}=10, \mathrm{Kp}=1.0$, and $\gamma=0.50$ with time $\tau=10$. These values are critiqued as homogeneous throughout the present study in respective figures.

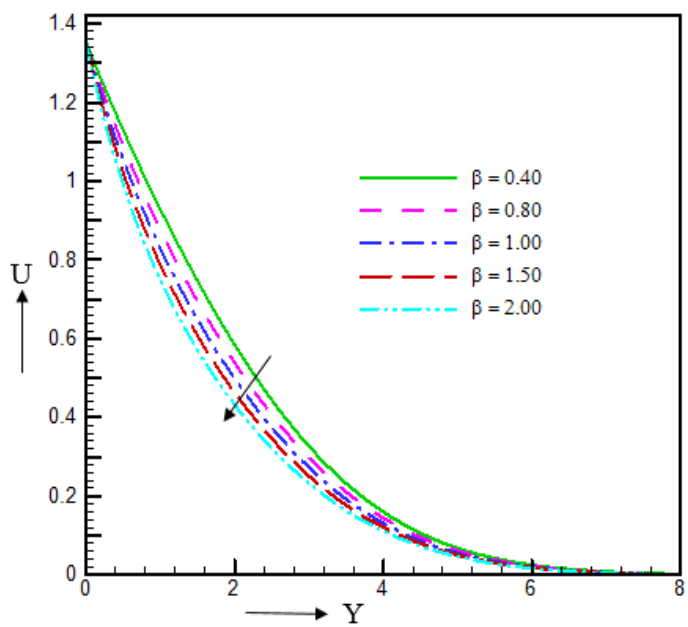

Figure 2. The illustration of $\beta$ on $U$

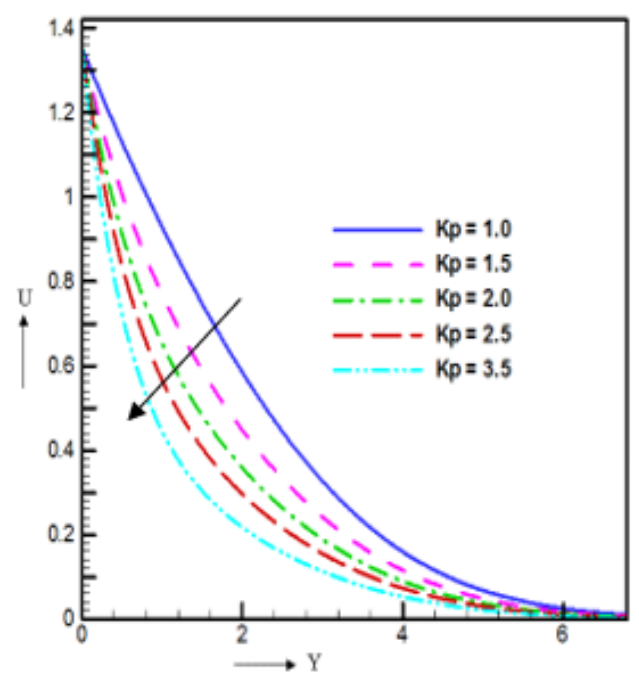

Figure 3. The illustration of $\mathrm{Kp}$ on $\mathrm{U}$

The impression of separate values of $\beta$ and $\mathrm{Kp}$ on velocity are elucidated in Figure 2 and Figure 3, respectively. It is 
proclaimed that velocity are economized $13.84 \%, 9.01 \%$, $20.8 \%$, and $19.92 \%$ by $\beta=0.40$ to $\beta=0.80, \beta=0.80$ to $\beta=1.00$, $\beta=1.00$ to $\beta=1.50$ and $\beta=1.50$ to $\beta=2.00$ at $Y=0.4556$. Physically, by elaborating the values of $\beta$ which remises the yield stress suppresses in the fluid velocity. Also, velocity is extenuation due to the prolongation of $\mathrm{Kp}$ which ensues in Figure 3. Naturally, permeability of porous medium which propagated a resistive force in the fluid. The numerical evolution of isolated parameters of $U$ are flaunted in the Table 1 and Figures 2-3.

Table 1. Delineates the curve to curve fluid velocity (Figures. 2, 3) at $\mathrm{Y}=0.4556$ and $\tau=10$

\begin{tabular}{ccc}
\hline $\begin{array}{c}\text { Values of } \\
\text { Parameter }\end{array}$ & $\begin{array}{c}\text { Numerical } \\
\text { Value }\end{array}$ & $\begin{array}{c}\text { Increase/Decrease in } \\
(\%)\end{array}$ \\
\hline$\beta=0.40$ & 0.13561 & Initial position \\
$\beta=0.80$ & 0.11684 & $13.84 \%$ decrease \\
$\beta=1.00$ & 0.10632 & $9.01 \%$ decrease \\
$\beta=1.50$ & 0.08413 & $20.87 \%$ decrease \\
$\beta=2.00$ & 0.06737 & $19.92 \%$ decrease \\
\hline $\mathrm{Kp}=1.00$ & 0.13561 & Initial position \\
$\mathrm{Kp}=1.50$ & 0.10002 & $26.24 \%$ decrease \\
$\mathrm{Kp}=2.00$ & 0.07755 & $22.47 \%$ decrease \\
$\mathrm{Kp}=2.50$ & 0.06311 & $18.62 \%$ decrease \\
$\mathrm{Kp}=3.50$ & 0.04589 & $27.29 \%$ decrease \\
\hline
\end{tabular}

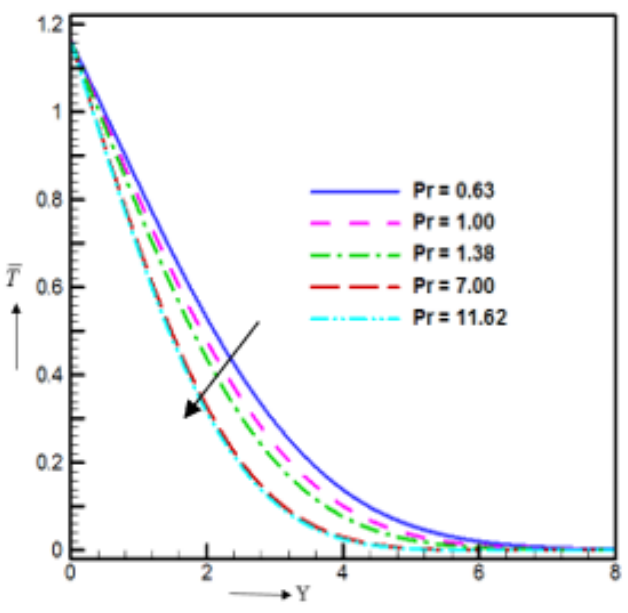

Figure 4. The illustration of $\operatorname{Pr} \bar{T}$

The impression of $\mathrm{Pr}, \mathrm{Nb}, \mathrm{Ra}$ and $\mathrm{S}$ on temperature schemes are found in the Figures 4-7. It is worth mentioned that temperature allocations are collapsed due to the enhancement of Pr. Instinctively, high Prandtl number have higher viscosity, which constricted the thermal boundary layer depth. Also, the temperature delineations are retrenched considerable with the accrual of $\mathrm{Nb}$ because it innovate an unbridled motions in the fluid. Here, temperature depictions are protracted as $35.46 \%, 16.02 \%$, $9.84 \%$, and $7.06 \%$ for $\mathrm{Ra}=0.50$ to $\mathrm{Ra}=1.00, \mathrm{Ra}=1.00$ to $\mathrm{Ra}=1.50, \mathrm{Ra}=1.50$ to $\mathrm{Ra}=2.00$ and $\mathrm{Ra}=2.00$ to $\mathrm{Ra}=2.50$ because the thermal boundary layer thickness are prosecuted due to completion of Ra. Further, temperature disbursements are surged due to augmentation of $\mathrm{S}$. The preeminent vindication behind this upshot is that the heat absorption invokes a drop in the kinetic energy as well as thermal energy of the fluid. The numerical disposition of relative parameters are evinced in the Table 2 and Figures 4-7.

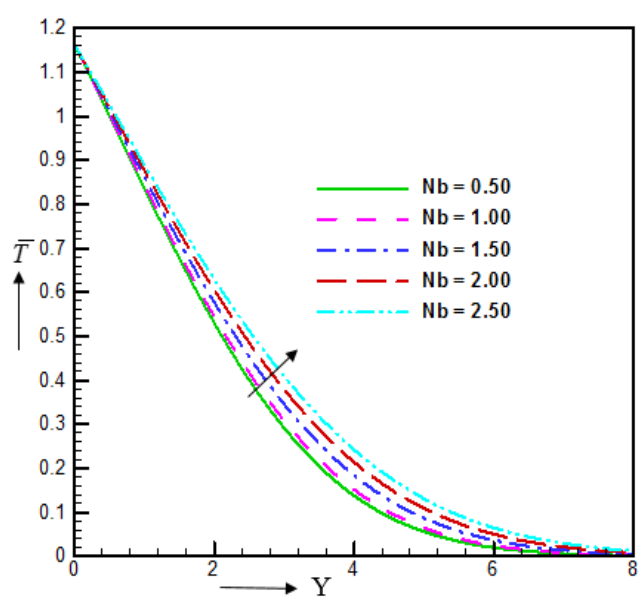

Figure 5. The illustration of $\mathrm{Nt}$ on $\bar{T}$

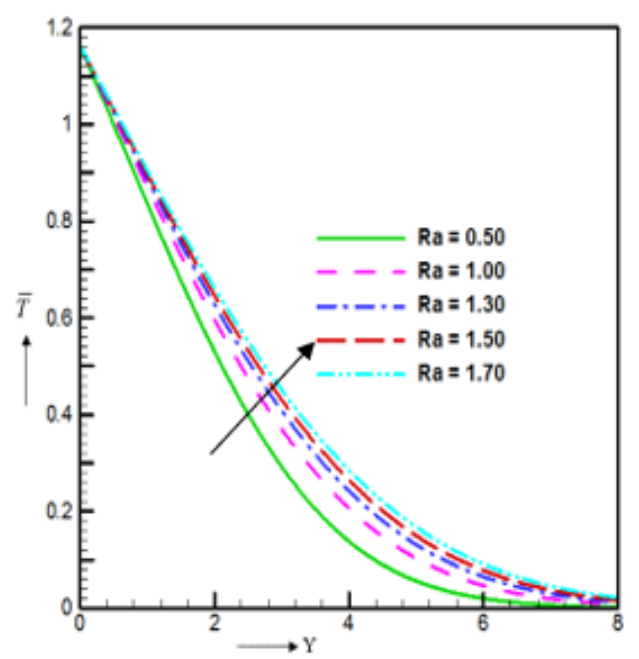

Figure 6. The illustration of $\mathrm{Ra}$ on $\bar{T}$

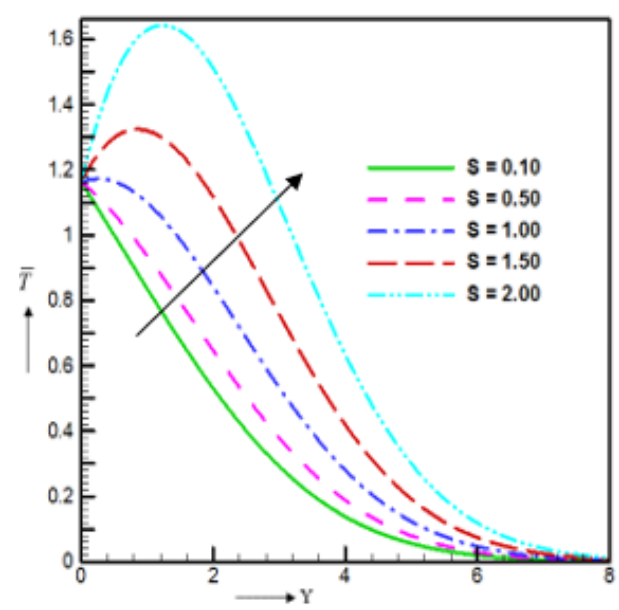

Figure 7. The illustration of $\mathrm{S}$ on $\bar{T}$

The denouements of $\gamma, \mathrm{Sc}$, Le and $\mathrm{Nb}$ on concentration propagations are eliciting in Figures 8-11. By the offshoot of chemical reaction, concentration is alleviated because the positive values of chemical reaction parameter $(\gamma>0)$ presages the sequels of destructive chemical reaction on the concentration field. It is fact that, Sc and Le constricts the concentration. It is true by physically that Schmidt number 
aggrandizes the density of the fluid as well as concentration buoyancy force in the fluid. Also, from Figure 11, we perceived that concentration disposals abridge to the amplifications of $\mathrm{Nb}$ which leads the concentration allocation. Physically, it is intimated that $\mathrm{Nt}$ elongates the nanoparticle diffusion. The numerical values of these are dispensed in the Table 3 and Figures 8-11.

The Figures 12 to 17 epitomize the repercussions of $\beta, \mathrm{Ra}$, $\mathrm{Gr}$ and Pr on skin friction coefficient $\left(\mathrm{C}_{\mathrm{f}}\right)$, Nusselt number $\left(\mathrm{N}_{\mathrm{u}}\right)$ and Sherwood number $\left(\mathrm{S}_{\mathrm{h}}\right)$ for Casson nanofluid. It is devised that, $C_{f}$ protracts with the accession of $\beta$ and $\gamma$ which is disported in Figure 12 and Figure 13 respectively. Physically, skin friction is aggrandized the rate of velocity of the fluid flow. On the other hand the Nusselt number is a decay function of $\mathrm{Ra}$ and $\mathrm{Gr}$ but proliferation function of Pr. Generally, convective heat transfer distends with the quadrupling of Nusselt number. Also it is articulated from Figure 17 that, the Sherwood number prolongs with the retrenchment of Grashof number (Gr).

Table 2. Incarnates the curve to curve fluid temperature (Figures 4-7) at $\mathrm{Y}=4.18060$ and $\tau=10.0$

\begin{tabular}{ccc}
\hline $\begin{array}{c}\text { Values of } \\
\text { Parameter }\end{array}$ & $\begin{array}{c}\text { Numerical } \\
\text { Value }\end{array}$ & $\begin{array}{c}\text { Increase/Decrease in } \\
(\%)\end{array}$ \\
\hline $\mathrm{Pr}=0.63$ & 0.11834 & Initial position \\
$\mathrm{Pr}=1.00$ & 0.10988 & $7.14 \%$ decrease \\
$\mathrm{Pr}=1.38$ & 0.09093 & $17.24 \%$ decrease \\
$\mathrm{Pr}=7.00$ & 0.05362 & $41.03 \%$ decrease \\
$\mathrm{Pr}=11.62$ & 0.05030 & $6.19 \%$ decrease \\
\hline $\mathrm{Ra}=0.50$ & 0.11834 & Initial position \\
$\mathrm{Ra}=1.00$ & 0.18336 & $35.46 \%$ increase \\
$\mathrm{Ra}=1.50$ & 0.21834 & $16.02 \%$ increase \\
$\mathrm{Ra}=2.00$ & 0.24216 & $9.84 \%$ increase \\
$\mathrm{Ra}=2.50$ & 0.26056 & $7.06 \%$ increase \\
\hline $\mathrm{Nb}=0.50$ & 0.11834 & Initial position \\
$\mathrm{Nb}=1.00$ & 0.13127 & $9.85 \%$ increase \\
$\mathrm{Nb}=1.50$ & 0.16192 & $18.93 \%$ increase \\
$\mathrm{Nb}=2.00$ & 0.19097 & $15.31 \%$ increase \\
$\mathrm{Nb}=2.50$ & 0.21830 & $12.52 \%$ increase \\
\hline $\mathrm{S}=0.10$ & 0.11834 & Initial position \\
$\mathrm{S}=0.50$ & 0.16319 & $27.48 \%$ increase \\
$\mathrm{S}=1.00$ & 0.24429 & $33.19 \%$ increase \\
$\mathrm{S}=1.50$ & 0.36841 & $33.79 \%$ increase \\
$\mathrm{S}=2.00$ & 0.56075 & $34.33 \%$ increase \\
\hline
\end{tabular}

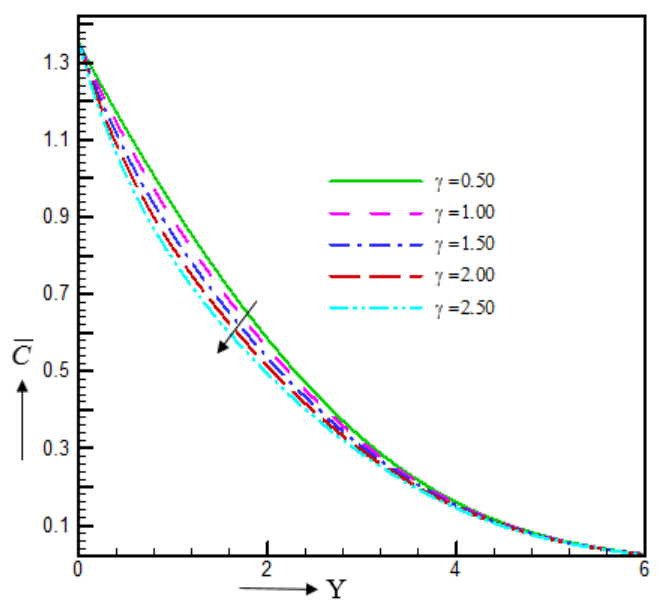

Figure 8. The illustration of $\lambda$ on $\bar{C}$

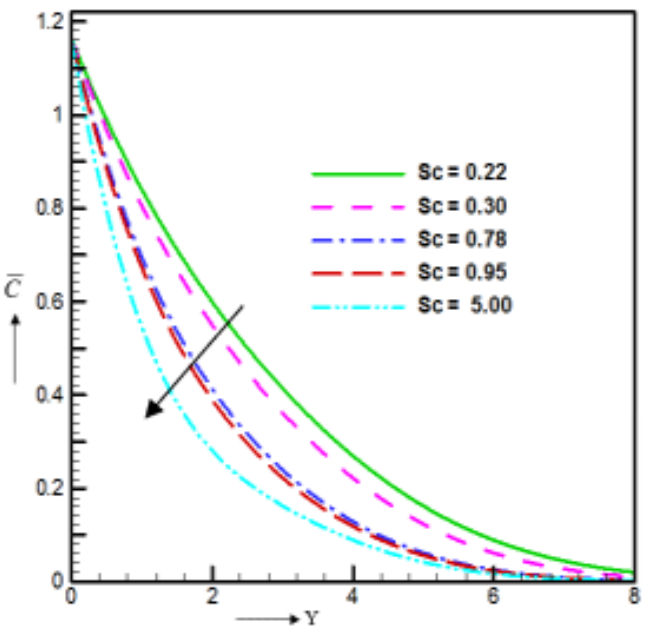

Figure 9. The illustration of Sc on $\bar{C}$

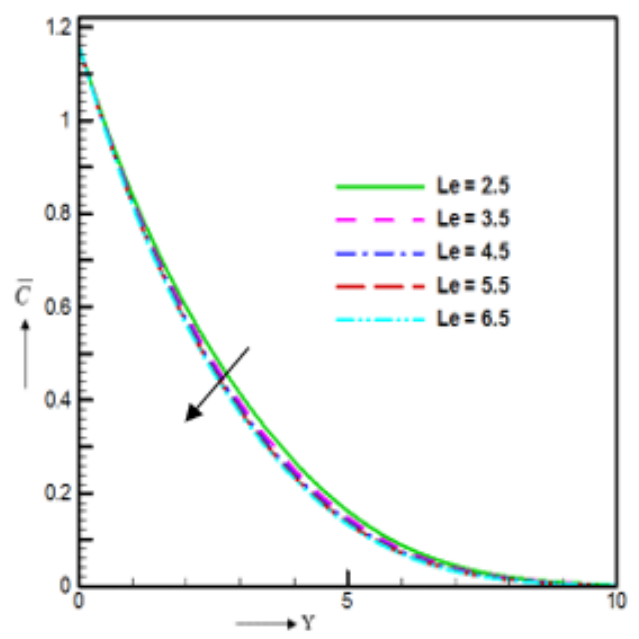

Figure 10. The illustration of Le on $\bar{C}$

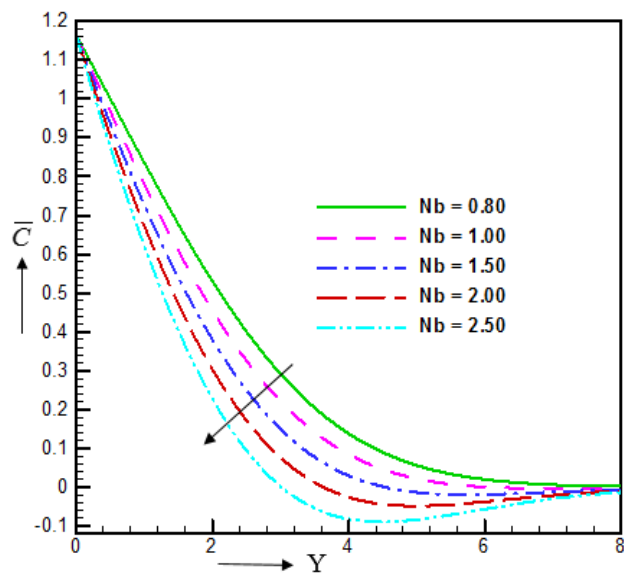

Figure 11. The illustration of $\mathrm{Nt}$ on $\bar{C}$

Table 3. Emblematizes the curve to curve fluid concentration (Figures 8-11) at $\mathrm{Y}=4.18060$ and $\tau=10.0$

\begin{tabular}{ccc}
\hline $\begin{array}{c}\text { Values of } \\
\text { parameter }\end{array}$ & $\begin{array}{c}\text { Numerical } \\
\text { value }\end{array}$ & $\begin{array}{c}\text { Increase/decrease in } \\
(\%)\end{array}$ \\
\hline$\gamma=0.50$ & 0.24736 & Initial position \\
$\gamma=1.00$ & 0.19056 & $22.96 \%$ decrease \\
$\gamma=1.50$ & 0.14917 & $21.72 \%$ decrease
\end{tabular}




\begin{tabular}{ccc}
$\gamma=2.00$ & 0.11864 & $20.47 \%$ decrease \\
$\gamma=2.50$ & 0.09583 & $19.22 \%$ decrease \\
\hline $\mathrm{Le}=2.50$ & 0.24736 & Initial position \\
$\mathrm{Le}=3.50$ & 0.23075 & $6.71 \%$ decrease \\
$\mathrm{Le}=4.50$ & 0.22136 & $4.06 \%$ decrease \\
$\mathrm{Le}=5.50$ & 0.21532 & $2.73 \%$ decrease \\
$\mathrm{Le}=6.50$ & 0.21111 & $1.96 \%$ decrease \\
\hline $\mathrm{Sc}=0.22$ & 0.24736 & Initial position \\
$\mathrm{Sc}=0.30$ & 0.20128 & $33.97 \%$ decrease \\
$\mathrm{Sc}=0.78$ & 0.11386 & $18.62 \%$ decrease \\
$\mathrm{Sc}=0.95$ & 0.10479 & $43.43 \%$ decrease \\
$\mathrm{Sc}=5.00$ & 0.07891 & $24.79 \%$ decrease \\
\hline $\mathrm{Nb}=0.80$ & 0.24736 & Initial position \\
$\mathrm{Nb}=1.00$ & 0.20355 & $17.71 \%$ decrease \\
$\mathrm{Nb}=1.80$ & 0.19804 & $2.71 \%$ decrease \\
$\mathrm{Nb}=2.00$ & 0.18124 & $8.48 \%$ decrease \\
$\mathrm{Nb}=2.50$ & 0.15329 & $15.82 \%$ decrease \\
\hline
\end{tabular}

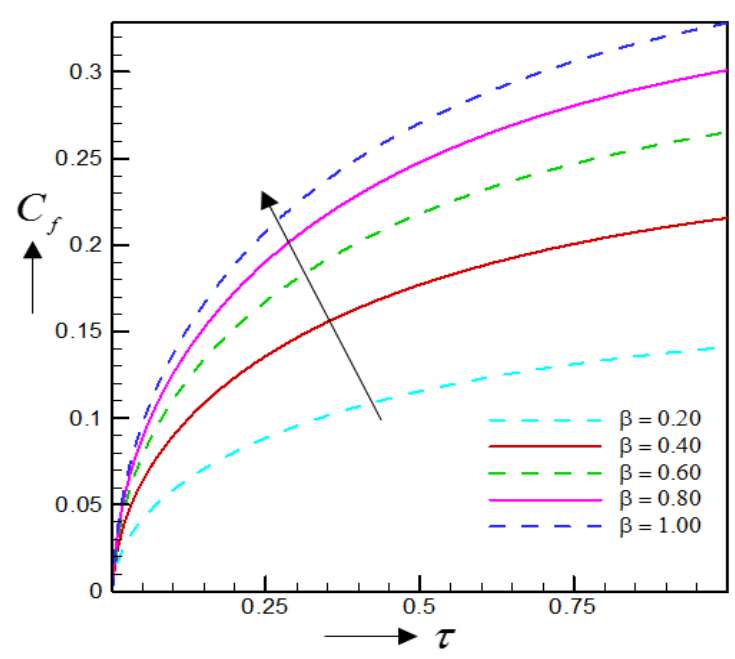

Figure 12. Skin friction for different values of $\beta$

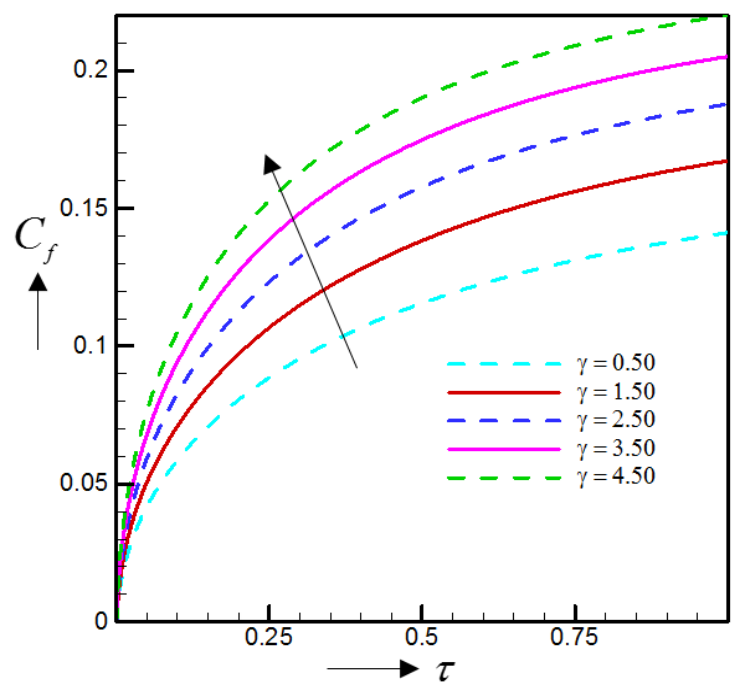

Figure 13. Skin friction for different values of $\gamma$

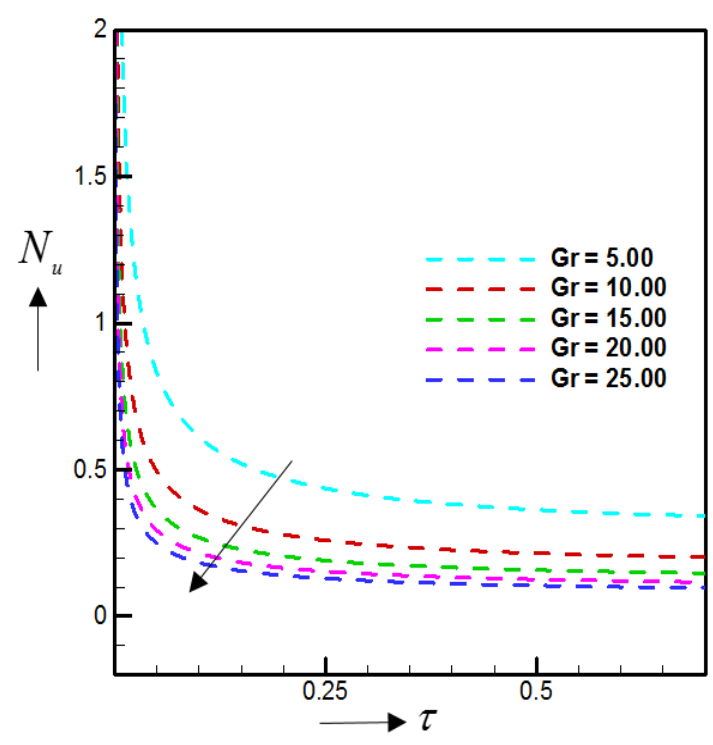

Figure 14. Nusselt number for different values of $\mathrm{Gr}$

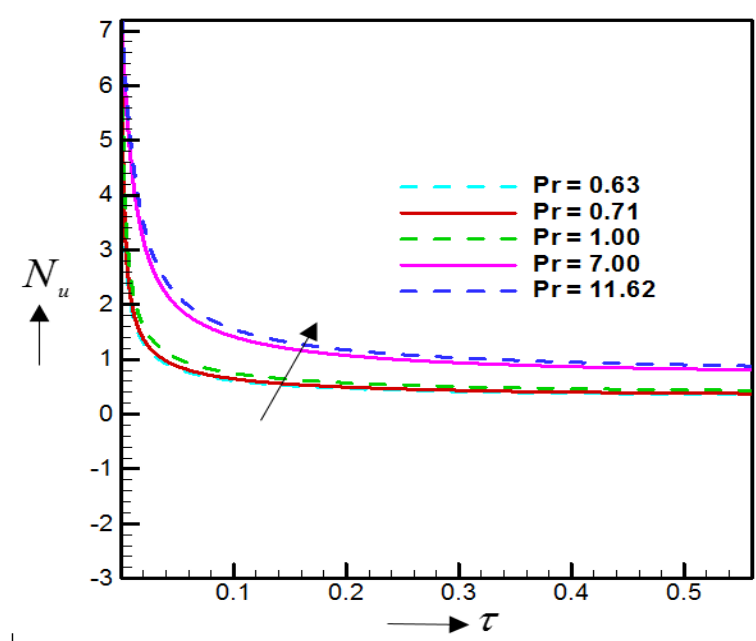

Figure 15. Nusselt number for different values of $\mathrm{Pr}$

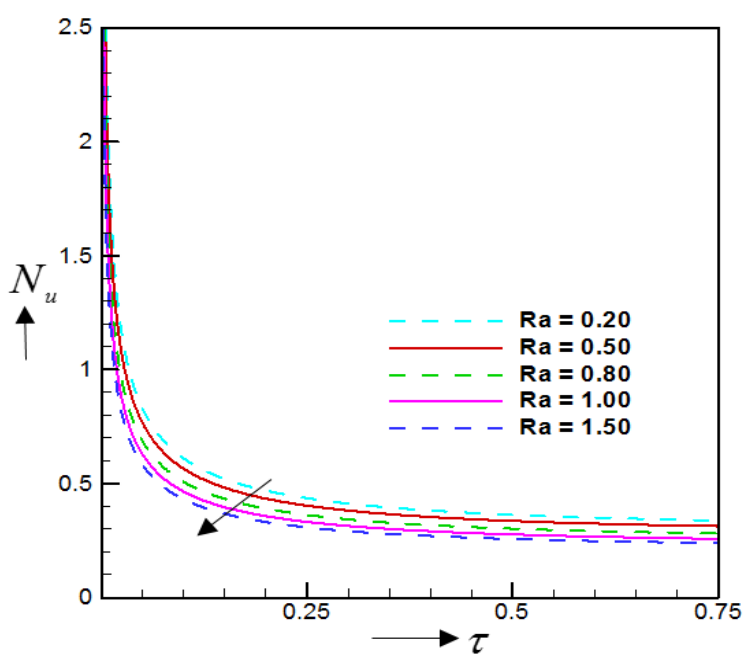

Figure 16. Nusselt number for different values of $\mathrm{Ra}$ 


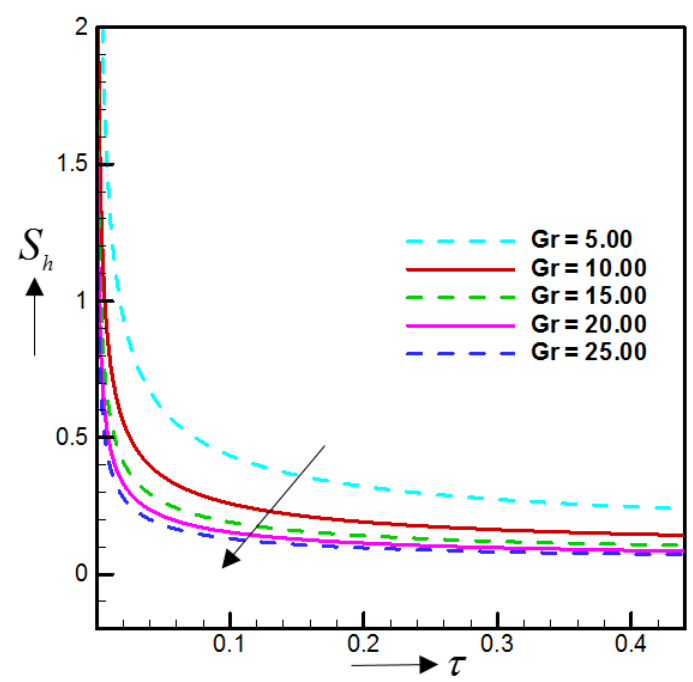

Figure 17. Sherwood number for different values of Gr

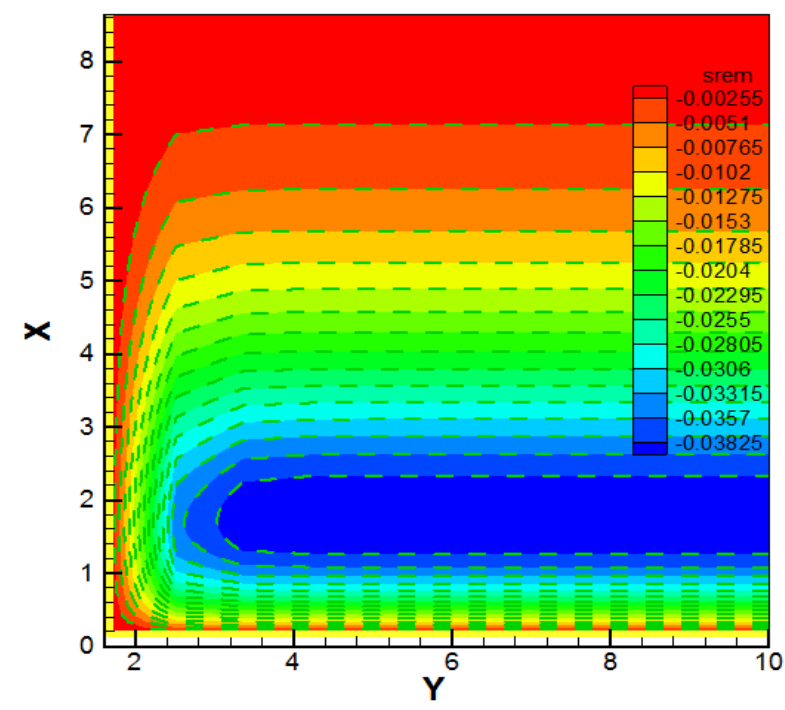

Figure 18. Illustration of Streamlines for $\mathrm{Ra}=0.50$ and $\mathrm{Ra}=1.50$

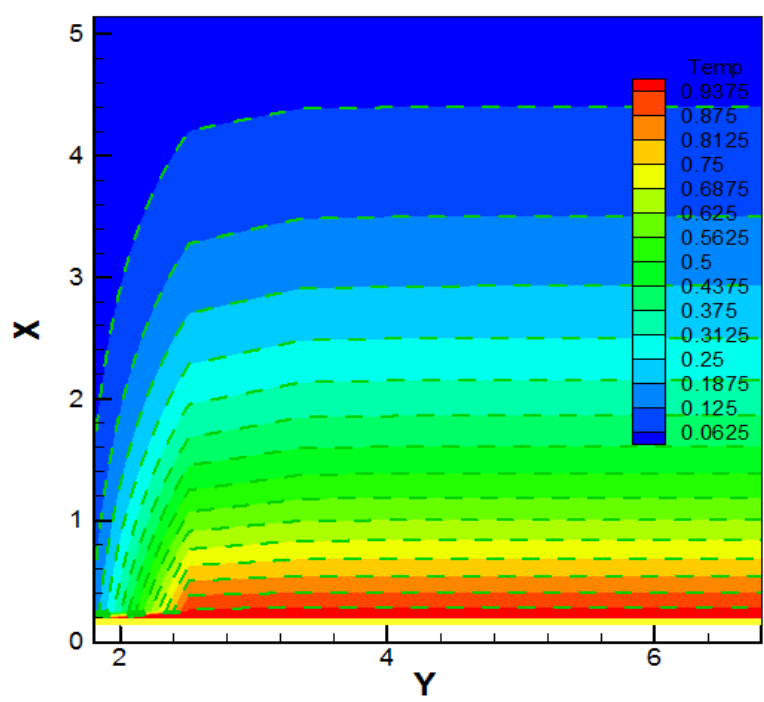

Figure 19. Illustration of Isotherms for $\mathrm{Ra}=0.50$ and $\mathrm{Ra}=1.50$

The dimensionless equations are unravelled numerically by EFDM such that, $\mathrm{X}$ and $\mathrm{Y}$ axis are dimensionless which depicts the mash point. Also, the stream and isotherms curves are disported in the above prominent Figures. Here, legend values of stream and isotherms insinuate the contours levels. Furthermore, streamlines dispensations can be utilized to meliorate the visualization of fluid fields. The boundary layer system of change can be proclaimed by an isotherm, where the temperature endures constant $(\Delta \mathrm{T}=0)$. An isotherm at $0{ }^{\circ} \mathrm{C}$ is denominated the freezing level. The denouement of radiation parameter $(\mathrm{Ra})$ on streamlines and isotherms are construed in Figure 18 and Figure 19. The momentum boundary layer and thermal boundary layer inflate due to the escalation of Ra. Also, Figure 19 evinces the thermal direction of fluid with the comportment of two distinct thermal radiation parameters. The isotherms plot of the momentum boundary layer thickness with the poise of $\mathrm{Nb}$ where the green dashed flood lines is for $\mathrm{Nb}=0.50$ and $\mathrm{Nb}=2.50$ is trumped in Figure 20. Figure 21 struts the contour flood view for thermal Brownian motion parameter difference. The legend values of Figure 21 are given in the contours levels. Here we perceived that the thermal and momentum boundary layer thickness is augmented due to addendum of $\mathrm{Nb}$.

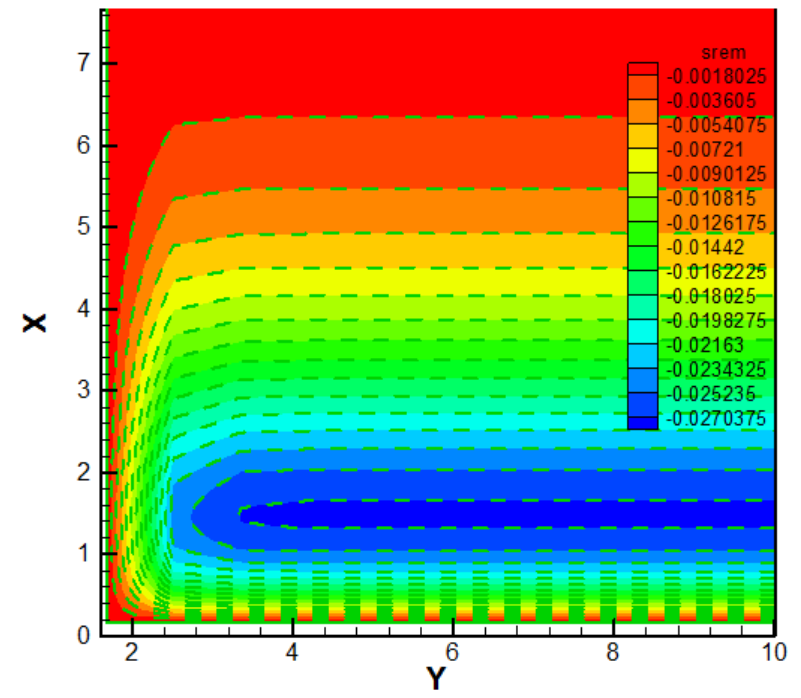

Figure 20. Illustration of Streamlines for $\mathrm{Nb}=0.50$ and $\mathrm{Nb}=2.50$

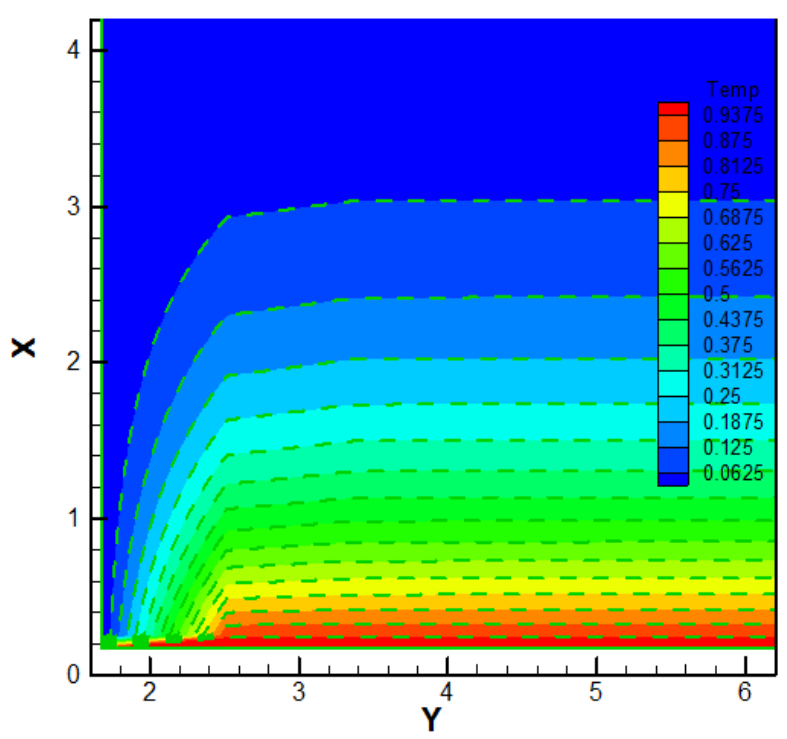

Figure 21. Illustration of Isotherms for $\mathrm{Nb}=0.50$ and $\mathrm{Nb}=2.50$ 


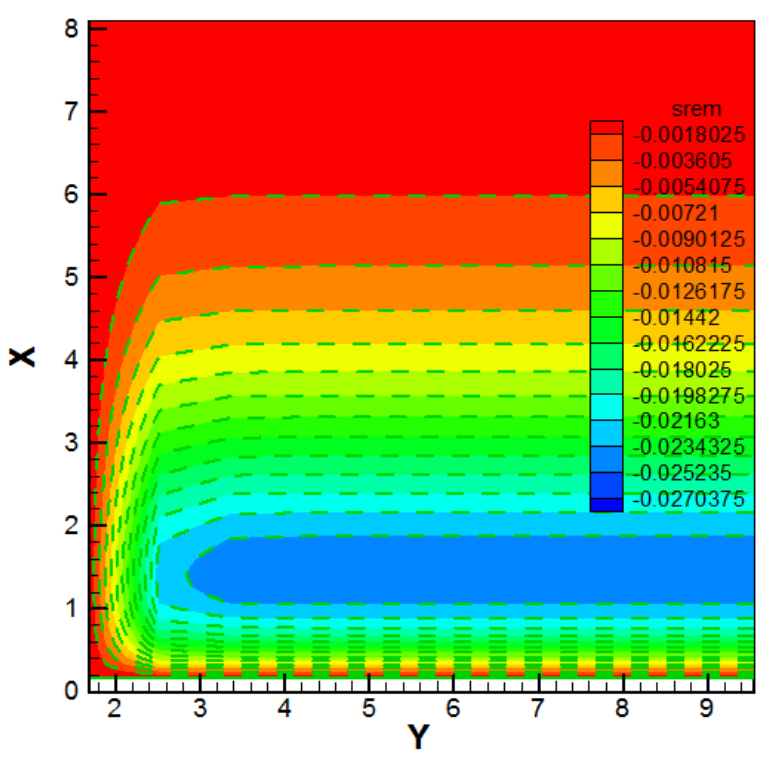

Figure 22. Illustration of Streamlines for $\mathrm{Nt}=0.80$ and $\mathrm{Nt}=1.30$

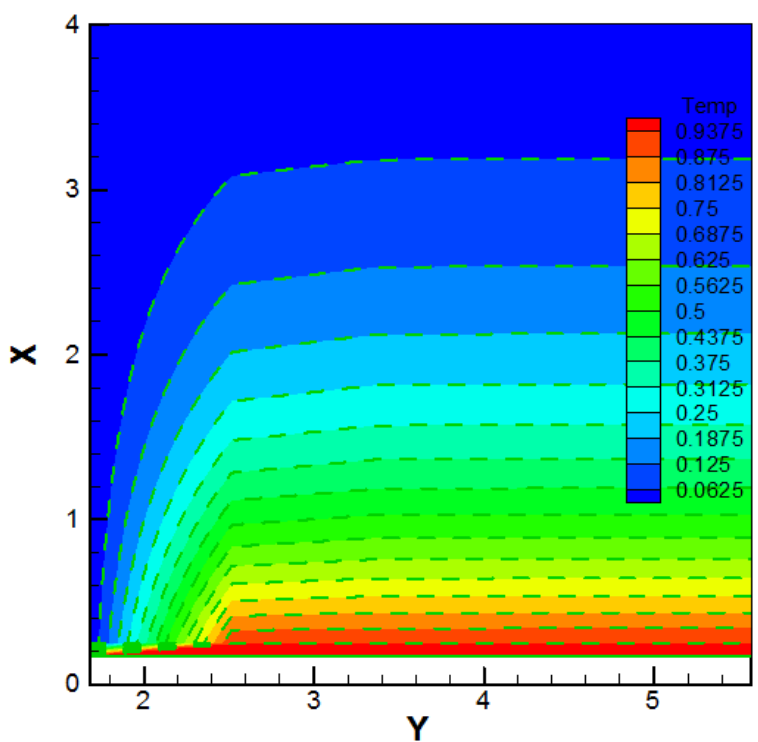

Figure 23. Illustration of Isotherms for $\mathrm{Nt}=0.80$ and $\mathrm{Nt}=1.30$

The momentum and thermal direction of fluid (thermal boundary layer thickness) with divergent $\mathrm{Nt}$ are flaunted in the Figure 22 and 23. Figure 22 divulges the contour flood view of momentum boundary layer and Figure 23 imparts the thermal boundary layer thickness with legend values which are the contours levels. In this apprehension we have discerned that the momentum boundary layer thickness is escalating but thermal boundary layer is abbreviating due to the propagation of Nt.

\section{CONCLUSION}

In this disquisition, the unsteady MHD heat and mass transfer of Casson nanofluid flow in the appearance of variable thermal conductivity, heat absorption and thermal radiation has been propounded. The predicament is evaluated under the influence of strong cross field and consideration has been endorsed to those pragmatic fluids which are altered as liquid metals. The governing model equations are elucidated by utilizing explicit finite difference method (EFDM) and the numerical results are enumerated for the divergent dimensionless parameters. In this observation the Casson parameter and permeability of porous medium abates the velocity profiles. The Nusselt number is plunged by radiation parameter and Grashof number but accumulated by Prandtl number. Also, the skin friction coefficient proliferates with the inflation of Casson parameter and chemical reaction parameter. Further, Brownian motion parameter $(\mathrm{Nb})$, chemical reaction $(\gamma)$, Schmidt number (Sc) and Lewis number (Le) wanes the concentration profiles. But, Brownian motion parameter $(\mathrm{Nb})$ which elevates the temperature profiles. Furthermore, temperature depictions are aggravating due to the amplification of heat source parameter and radiation parameter but dwindling due to the amelioration of Prandtl number.

Table 4. The previous results by Ghadikolaei et al. [12]

\begin{tabular}{ccccccc}
\hline \multicolumn{6}{c}{ Previous Results by Ghadikolaei et al. [12] } \\
\hline $\begin{array}{c}\text { Increased } \\
\text { Parameters }\end{array}$ & $\mathbf{U}$ & $\overline{\mathbf{T}}$ & $\overline{\mathbf{C}}$ & $\begin{array}{c}\text { Skin } \\
\text { fri. }\end{array}$ & $\begin{array}{c}\text { Nusl. } \\
\text { Num. }\end{array}$ & $\begin{array}{c}\text { Sher. } \\
\text { Num. }\end{array}$ \\
\hline $\mathrm{Gr}$ & & & & & Inc & \\
$\mathrm{Nb}$ & & Inc & Dec & & & \\
$\beta$ & Dec & Inc & Inc & & & \\
$\mathrm{S}$ & & Inc & & Inc & & \\
$\mathrm{R}$ & & Inc & & & Inc & \\
$\gamma$ & & & Dec & & & Inc \\
Pr & & Dec & & & & \\
Le & & & Dec & & & \\
\hline
\end{tabular}

Table 5. Comparison of the accuracy of the present results with the previous results by Ghadikolaei et al. [12]

\begin{tabular}{ccccccc}
\hline \multicolumn{6}{c}{ Previous Results by Ghadikolaei et al. [12] } \\
\hline $\begin{array}{c}\text { Increased } \\
\text { Parameters }\end{array}$ & $\mathbf{U}$ & $\overline{\mathbf{T}}$ & $\overline{\mathbf{C}}$ & $\begin{array}{c}\text { Skin } \\
\text { fri. }\end{array}$ & $\begin{array}{c}\text { Nusl. } \\
\text { Num. }\end{array}$ & $\begin{array}{c}\text { Sher. } \\
\text { Num. }\end{array}$ \\
\hline $\mathrm{Gr}$ & & & & & & \\
$\mathrm{Nb}$ & & Inc & Dec & & & \\
$\beta$ & Dec & & & & & \\
$\mathrm{S}$ & & Inc & & Inc & & \\
$\mathrm{R}$ & & Inc & & & Dec & \\
$\gamma$ & & & Dec & & & \\
Pr & & Dec & & & & \\
Le & & & Dec & & &
\end{tabular}

Comparison of the accuracy of the present results with the previous results by Ghadikolaei et al. [12] are impersonated in the above Table 4. Here, it is permitted that, all values are same without just only one result is isolated with the works of Ghadikolaei et al. [12]. In this present disquisition, Nusselt number is dwindled by radiation parameter but opposite with the work of Ghadikolaei et al. [12].

\section{REFERENCES}

[1] Oyelakin IS, Mondal S, Sibanda P. (2016). Unsteady Casson nanofluid flow over a stretching sheet with thermal radiation, convective and slip boundary conditions. Alexandria Engineering Journal 55: 10251035. https://doi.org/10.1016/j.aej.2016.03.003

[2] Kataria HR, Patel HR. (2016). Soret and heat generation effects on MHD Casson fluid flow past an oscillating vertical plate embedded through porous medium. Alexandria Engineering Journal 55: 2125-2137. https://doi.org/10.1016/j.aej.2016.06.024 
[3] Ahmed N, Khan U, Khan SI, Bano S, Mohyud-Din ST. (2017). Effects on magnetic field in squeezing flow of a Casson fluid between parallel plates. Journal of King Saud University Science 29: 119-125. https://doi.org/10.1016/j.jksus.2015.03.006

[4] Mahanta G, Shaw S. (2016). 3D Casson fluid flow past a porous linearly stretching sheet with convective boundary condition. Alexandria Engineering Journal 54: 653-659. https://doi.org/10.1016/j.aej.2015.04.014

[5] Afikuzzaman M, Ferdows M, Alam MM. (2015). Unsteady MHD Casson fluid flow through a parallel plate with Hall current. Procedia Engineering 105: 287 293. https://doi.org/10.1016/j.proeng.2015.05.111

[6] Biswas R, Mondal M, Sarkar DR, Ahmmed SF. (2017). Effects of radiation and chemical reaction on MHD unsteady heat and mass transfer of Casson fluid flow past a vertical plate. Journal of Advances in Mathematics and Computer Science 23(2): 1-16. http://www.sciencedomain.org/review-history/19926

[7] Afikuzzaman M, Alam MM. (2016). MHD casson fluid flow through a parallel plate. Thammasat International Journal of Science and Technology 21(1): 1-7. https://tcithaijo.org/index.php/SciTechAsia/52122

[8] Wahiduzzaman M, Islam MT, Sultana P, Afikuzzaman M. (2014). MHD couette flow of a Casson fluid between parallel porous plates. Progress in Nonlinear Dynamics and Chaos 2(2): 51-60.

[9] Biswas R, Ahmmed SF. (2018). Effects of Hall current and chemical reaction on MHD unsteady heat and mass transfer of Casson nanofluid flow through a vertical plate. Journal of Heat Transfer 140: 092402-1.

[10] Khalid A, Khan I, Khan A, Shafie S. (2015). Unsteady MHD free convection flow of casson fluid past over an oscillating vertical plate embedded in a porous medium. Engineering Science and Technology 18: 309-317. https://doi.org/10.1016/j.jestch.2014.12.006

[11] Ali ME, Sandeep N, (2017). Cattaneo-christov model for radiative heat transfer of magneto-hydrodynamic cassonferrofluid: A numerical study. Results in Physics 7: 2130. https://doi.org/10.1016/j.rinp.2016.11.055

[12] Ghadikolaei SS, Hosseinzadeh KH, Ganji DD, Jafari B. (2018). Nonlinear thermal radiation effect on magneto Casson nanofluid flow with Joule heating effect over an inclined porous stretching sheet. Case Studies in Thermal Engineering 12:

176-187. https://doi.org/10.1016/j.csite.2018.04.009

[13] Chai YH, Yusup S, Soonc CV, Arpinc MT. (2016). Rheological behaviour of graphene Nano-sheets in hydrogenated oil-based drilling fluid. Procedia Engineering Journal 148: 49-56. https://doi.org/10.1016/j.proeng.2016.06.490

[14] Ganga B, Ansari SMY, Ganeshc NV, Hakeem AKA. (2016). MHD flow of Boungiorno model nanofluid over a vertical plate with internal heat generation/absorption. Propulsin and Power Research 5(3): 211-222. https://doi.org/10.1016/j.jppr.2016.07.003

[15] Khan NA, Sultan F, Rubbab Q. (2015). Optimal solution of nonlinear heat and mass transfer in a two-layer flow with nano-Eyring-Powell fluid. Results in Physics 5: 199-205. https://doi.org/10.1016/j.rinp.2015.08.006

[16] Biswas P, Arifuzzaman SM, Karim I, Khan MS. (2017). Impacts of magnetic field and radiation absorption on mixed convective Jeffrey nano fluid flow over a vertical stretching sheet with stability and convergence analysis.
Journal of Nanofluid 6(6):

1082-1095. https://doi.org/10.1166/jon.2017.1407

[17] Bég OA, Khan MS, Karim I, Alam MM., Ferdows M. (2014). Explicit numerical study of unsteady hydromagnetic mixed convective nanofluid flow from an exponentially stretching sheet in porous media. Applied Nanoscience 4: 943-957.

[18] Dogonchi AS, Divsalar K, Ganji DD. (2016). Flow and heat transfer of MHD nanofluid between parallel plates in the presence of thermal radiation. Computer Methods in Applied Mechanics and Engineering 310: 58-76. https://doi.org/10.1016/j.cma.2016.07.003

[19] Dogonchi AS, Alizadeh M, Ganji DD. (2017). Investigation of MHD go-water nanofluid flow and heat transfer in a porous channel in the presence of thermal radiation effect. Advanced Powder Technology 28(7): 1815-1825. https://doi.org/10.1016/j.apt.2017.04.022

[20] Ahmmed SF, Biswas R, Afikuzzaman M. (2018). Unsteady magnetohydrodynamic free convection flow of nanofluid through an exponentially accelerated inclined plate embedded in a porous medium with variable thermal conductivity in the presence of radiation. Journal of Nanofluids 7: 891-901. https://doi.org/10.1166/jon.2018.1520

[21] Leekea GA, Tiejun L, Bridsona RH, Jonathan PKS. (2016). Application of nano-particle coatings to carrier particles using an integrated fluidized bed supercritical fluid precipitation process. Journal of Supercritical Fluids 91: 7-14. https://doi.org/10.1016/j.supflu.2014.03.012

[22] Uddin MJ, Kalbani KSA, Rahman MM, Alam MS, Eltayed IA. (2016). Fundamentals of nanofluids: evolution, applications and new theory. Official Journal of Biomathematical Society of India 2(1): 1-32.

[23] Bilal S, Rehman KU, Malik MY, Hussain A, Awais M. (2017). Effect logs of double diffusion on MHD Prandtl nanofluid adjacent to 4 stretching surface by way of numerical approach. Results in Physics 7: 470-479. https://doi.org/10.1016/j.rinp.2016.11.008

[24] Arani AG, Zarei MS. (2015). Nonlocal vibration of yshaped CNT conveying nano-magnetic viscous fluid under magnetic field. Ain Shams Engineering Journal 6: 565-575. https://doi.org/10.1016/j.asej.2014.11.012

[25] Murthy MVR, Raju RS, Rao JA. (2015). Heat and mass transfer effects on MHD natural convective flow past an infinite vertical porous plate with thermal radiation and Hall current. Procedia Engineering 127: 1330-1337. https://doi.org/10.1016/j.proeng.2015.11.491

[26] Das S, Jana RN, Makinde OD. (2015). Magnetohydrodynamic mixed convective slip flow over an inclined porous plate with viscous dissipation and joule heating. Alexandria Engineering Journal 54(2): 251-261. https://doi.org/10.1016/j.aej.2015.03.003

[27] Shit GC, Haldar R, Ghosh SK. (2016). Convective heat transfer and MHD viscoelastic nanofluid flow induced by a stretching sheet. International Journal of Applied and Computational Mathematics 2(59): 3-608.

[28] Ibrahim W. (2016). The effect of induced magnetic field and convective boundary condition on MHD stagnation point flow and heat transfer of upper-convected Maxwell fluid in the presence of nanoparticle past a stretching sheet. Propulsin and Power Research 5(2): 164-175. https://doi.org/10.1016/j.jppr.2016.05.003 
[29] Rani HP, Kim CN. (2010). A numerical study on unsteady natural convection of air with variable viscosity over an isothermal vertical cylinder. Korean Journal of Chemical Engineering 27: 759-765. https://doi.org/10.1007/s11814-010-0211-x

[30] Animasaun IJ, Oyem AO. (2014). Effect of variable viscosity, Dufour, soret and thermal conductivity on free convection heat and mass transfer of non-Darcian flow past porous flat surface. American Journal of Computational Mathematics 33: 271-292. https://doi.org/10.1016/j.jnnms.2014.10.008

[31] Lavanya B, Ratnam AL. (2014). Dufour and soret effects on steady MHD free convective flow past a vertical porous plate embedded in a porous medium with chemical reaction, radiation, heat generation and viscous dissipation. Advances in Applied Science and Research 5(1): 127-142.

[32] Khan N, Mahmood T, Sajid M, Hashmi MS. (2016). Heat and mass transfer on MHD mixed convection axisymmetric chemically reactive flow of Maxwell fluid driven by exothermal and isothermal stretching disks. International Journal of Heat and Mass Transfer 92: 1090-1105. https://doi.org/10.1016/j.ijheatmasstransfer.2015.09.001

[33] Tripathy RS, Dash GC, Mishra SR, Baag S. (2015). Chemical reaction effect on MHD free convective surface over a moving vertical plate through porous medium. Alexandria Engineering Journal 54: 673-679. https://doi.org/10.1016/j.aej.2015.04.012

[34] Das S, Guchhait SK, Jana RN, Makinde OD. (2016). Hall effects on an unsteady magneto- convection and radiative heat transfer past a porous plate. Alexandria Engineering Journal 55:

$1321-1331$ https://doi.org/10.1016/j.aej.2016.04.027

[35] Dogonchi AS, Ganji DD. (2016). Thermal radiation effect on the nano-fluid buoyancy flow and heat transfer over a stretching sheet considering Brownian motion. Journal of Molecular Liquids 223: 521-527. https://doi.org/10.1016/j.molliq.2016.08.090

[36] Hsiao KL. (2017). Combined electrical MHD heat transfer thermal extrusion system using Maxwell fluid with radiative and viscous dissipation effects. Applied Thermal Engineering 112: 1281-1288. https://doi.org/10.1016/j.applthermaleng.2016.08.208

[37] Biswas R, Afikuzzaman M, Mondal M, Ahmmed SF. (2018). MHD free convection and heat transfer flow through a vertical porous plate in the presence of chemical reaction. Frontiers in Heat and Mass Transfer 11: 13. https://doi.org/10.5098/hmt.11.13

[38] Ahmmed SF, Biswas R. (2019). Effects of radiation and chemical reaction on MHD unsteady heat and mass transfer of nanofluid flow through a vertical plate. Modelling Measurement and Control B 87(4): 213-220.

[39] Biswas R, Mondal M, Islam A. (2019). A steady MHD natural convection heat transfer fluid flow through a vertical surface in the existence of Hall current and radiation. Instrumentation, Mesure, Métrologie 2: 331356. https://i2m.revuesonline. com/accueil.jsp

\section{NOMENCLATURE}

\begin{tabular}{|c|c|}
\hline $\mathrm{x}, \mathrm{y}$ & Cartesian coordinates $(\mathrm{m})$ \\
\hline $\mathrm{u}, \mathrm{v}$ & Velocity components $(\mathrm{m} / \mathrm{s})$ \\
\hline$\gamma$ & Chemical reaction parameter $(-)$ \\
\hline $\mathrm{U}$ & Dimensionless primary velocity $(\mathrm{m} / \mathrm{s})$ \\
\hline Sc & Schmidt number (-) \\
\hline $\mathrm{Nu}$ & Nusselt number (-) \\
\hline $\mathrm{S}$ & Heat source parameter $(-)$ \\
\hline $\mathrm{Cf}$ & Skin friction (-) \\
\hline$\rho$ & Fluid density $\left(\mathrm{kg} / \mathrm{m}^{3}\right)$ \\
\hline$v$ & Kinematic viscosity $\left(\mathrm{m}^{2} \mathrm{~s}^{-1}\right)$ \\
\hline $\mathrm{Tm}$ & Mean fluid temperature $(\mathrm{K})$ \\
\hline Cs & Concentration susceptibility $(\mathrm{J} / \mathrm{kg} \cdot \mathrm{K})$ \\
\hline $\mathrm{Sh}$ & Sherwood number (-) \\
\hline $\bar{T}$ & Dimensionless fluid temperature $(\mathrm{K})$ \\
\hline $\mathrm{Nt}$ & Thermophoresis parameter (-) \\
\hline$\lambda$ & Thermal conductivity variation parameter (-) \\
\hline $\mathrm{g}$ & Acceleration due to gravity $\left(\mathrm{ms}^{-2}\right)$ \\
\hline M & Magnetic parameter $\left(\mathrm{Wbm}^{-2}\right)$ \\
\hline$\beta \mathrm{T}$ & Thermal expansion coefficient (-) \\
\hline $\operatorname{Pr}$ & Prandlt number (-) \\
\hline $\mathrm{Gr}$ & Grashof number (-) \\
\hline $\mathrm{Gm}$ & Modified Grashof number (-) \\
\hline $\mathrm{Kp}$ & Permeability of porous medium (-) \\
\hline$\beta$ & Casson parameter (-) \\
\hline $\mathrm{Sr}$ & Soret number (-) \\
\hline $\mathrm{T}$ & Temperature of fluid $(\mathrm{K})$ \\
\hline $\mathrm{k}$ & Thermal conductivity $(\mathrm{W} / \mathrm{m} \cdot \mathrm{K})$ \\
\hline $\mathrm{Cp}$ & Specific heat at constant pressure $\left(\mathrm{J} / \mathrm{m}^{3} \cdot \mathrm{K}\right)$ \\
\hline$\beta c$ & Concentration expansion coefficient (-) \\
\hline $\bar{C}$ & Dimensionless fluid concentration (-) \\
\hline Le & Lewis number (-) \\
\hline $\mathrm{Nb}$ & Brownian motion parameter (-) \\
\hline $\mathrm{Dm}$ & $\begin{array}{l}\text { Molecular diffusivity of the } \\
\text { concentration (-) }\end{array}$ \\
\hline & Stefan-Boltzmann constant $\left(\mathrm{W} / \mathrm{m}^{2} \mathrm{~K}^{4}\right)$ \\
\hline
\end{tabular}

